\title{
GLI OGGETTI DI UNA VOCE FEMMINILE AL VAGLIO DELL'ETICA DELL'AUTO-TRADUZIONE ASSISTITA: IL PAESE DOVE NON SI MUORE MAI DI ORNELA VORPSI
}

\section{A FEMALE VOICE ONTHE EXAMINATION OF THE ETHIC OF ASSISTED SELF-TRANSLATION: ORNELA VORPSI'S IL PAESE DOVE NON SI MUORE MAI}

\author{
Mario Rossi. Universität Wien - Austria \\ mario.rossi@univie.ac.at
}

\begin{abstract}
Riassunto Nel saggio si pone il problema della valutazione dell'autotraduzione in una prospettiva che analizza le molle che nel profondo muovono un testo letterario. Si analizza l'autotraduzione assistita del romanzo breve di Ornela Vorpsi Il paese dove non si muore mai dal francese all'italiano prendendo in considerazione la presenza di marche di genere in passi in cui siano presenti oggetti; la questione di genere percorre tutto il testo e quindi appare interessante verificare come questa si cristallizzi in significanti apparentemente poco permeabili alla modulazione denotativa e connotativa. Si giungerà alla conclusione che l'analisi dell'autotraduzione assistita può esser strumento valido per la definizione del grado di coerenza delle strutture profonde di un testo tanto più importante se la tematica è centrata sulla questione di genere.
\end{abstract}

Parole chiave: autotraduzione, questione di genere, connotazione e struttura profonda, oggetti, Ornela Vorpsi.

\begin{abstract}
This article approaches the problem of how to evaluate the significance of the critical feminist instances on an assisted self-translation text by applying the contrastive analysis method. The analysed work is $\mathrm{O}$. Vorpsi novel Il paese dove non si muore mai, which has been translated from Italian to French by M. Pozzoli, with the author's collaboration. The main aspect which will be analysed is the to investigate the presence of gender marks in passages which mention objects - informative and at the same time formative elements of the text-, since the gender issue crystallize in a particular way also in signifiers of an apparently low permeability to the connotative modulation, as it is the case for those signifiers related to objects. By contrasting the oscillations between the two texts, we will emphasize on the possible contradictions in the text's deep-lying structure. In this way, through the assisted self-translation analysis it can be measured to what extent the intended critical instances regarding the gender issue which are present throughout the novel are coherent and valid.
\end{abstract}

Keywords self-translation, gender, connotation and deep structure, objects, Ornela Vorpsi. 


\section{Introduzione}

Cosa concludere da questa serie di rivolgimenti? Lo confesso, io resto perplesso. Sono portato (...) a privilegiare l'entrata attraverso la porta dello straniero. Non siamo forse stati messi in movimento a causa del fatto della pluralità umana e dal doppio enigma dell'incomunicabilità tra gli idiomi e della traduzione malgrado tutto? E poi, senza la prova dello straniero, saremmo sensibili all'estraneità della nostra propria lingua? ${ }^{1}$

Narrazioni sono ideologiche nella loro configurazione e hanno conseguenze importanti in ciò che motiva e determina scelte etiche. (...) narrazioni sono dinamiche e aperte alla negoziazione. Poiché $\mathrm{i}$ traduttori sono impegnati nell'attraversamento e nella costruzione di narrazioni nella misura in cui negoziano differenti linguaggi e culture, questa struttura è produttiva, particolarmente dalla prospettiva di un attivo interesse rispetto al cambio di modi di pensiero finalizzati alla trasformazione di modi di essere e di intervenire nel mondo. ${ }^{2}$

Gli onesti quesiti coi quali Paul Ricoeur chiude Le paradigme de la traduction possono a buon diritto introdurre nella tortuosa storia editoriale dei testi letterari di O. Vorpsi. Può stupire la citazione da un filosofo che non si è dedicato alla teoria della traduzione in una prospettiva femminista. Il passo ci è sembrato interessante per l'inquietudine che testimonia e che percorre tutto il saggio del filosofo francese. D'altro canto si deve osservare che il taglio specifico del presente lavoro, vale a dire l'auto-traduzione assist ita di scrittura femminile osservata attraverso la lente puntata sugli oggetti ${ }^{3}$, non trova significative trattazioni nemmeno nel recente volume collett ivo Cordingley 2013 che annovera nomi prestigiosi come S. Bennett, R. Guldin, R. Grutman e J. Hokenson. La citazione dal saggio di Shread di teoria femminista della traduzione applicata a un caso concreto ci consente di giustificare in forma autorevole il nostro approccio che mira a rilevare la dimensione et ica presente nel processo autotraduttivo e estendibile a quello traduttivo; più precisamente cercheremo di individuare la dimensione et ica che può emergere nel rapporto tra due testi legati da pratica traduttiva ed esaminati nelle tensioni tra struttura di superficie e struttura profonda. Per far ciò cercheremo di valutare le interconnessioni tra ciò che la studiosa

\footnotetext{
1 Ricoeur, 2004, pp. 51-52.

2 Shread, 2011, p. 284 .

3 Lo sguardo filtrato attraverso l'attenzione per l'oggetto giustifica riferimenti bibliografici da discipline diverse: speriamo che si consideri il carattere eclettico della bibliografia come stimolo a percorrere sentieri poco battuti.
} 
chiama aspetti informativi e formativi del testo, intendendo, grosso modo, con il primo attributo il contenuto e con il secondo gli strumenti che danno forma al testo ${ }^{4}$.

Dopo questa premessa, volta a sgomberare il campo da frettolose riserve nei confronti delle nostre riflessioni, veniamo all'autrice di cui ci occuperemo e ai suoi oggetti. Migrata dall'Albania in Italia, dove dal 1991 ha frequentato I'Accademia di Belle Arti di Brera, Vorpsi, poliedrica art ista e scrittrice che può esser rubricata sotto la categoria della letteratura postcoloniale italiana, ha scritto presumibilmente in una prima lingua straniera, I'italiano approfondito durante il suo soggiorno lombardo, e ha pubblicato in prima edizione la traduzione dei suoi primi quattro testi letterari in una seconda lingua straniera, il francese, con la quale ha avuto contatto quotidiano dopo il 1998, anno in cui si è trasferita in Francia dove ha frequentato I'Università Paris VII ${ }^{5}$. La videoartista albanese ha pubblicato in ordine cronologico dal 2003 ad oggi i seguent $i$ test i di carattere narrativo: Il paese dove non si muore mai, Vetri rosa, La mano che non mordi, Bevete cacao van Houten!, Fuorimondo ${ }^{6}$, Tu convoiteras ${ }^{7}$. La versione francese del romanzo d'esordio è stata accolta presso Babel, mentre i seguenti tre testi sono apparsi, in francese, presso Actes Sud. Tutti i testi in italiano, se si eccettua Bevete cacao va Houten, hanno visto la luce dopo brevissimi intervalli di tempo rispetto alla pubblicazione della traduzione in francese ${ }^{8}$. I testi sono stati resi in francese prevalentemente da donne e sempre da persone diverse. Eccone l'elenco nell'ordine cronologico di apparizione delle edizioni francesi: Margherite Pozzoli traduce nel 2004 Il paese dove non si muore mai col titolo Le pays où l'on ne meurt jamais, apparso in italiano nel 2005 presso Einaudi; I'anno seguente Marianne Véron lavora su Bevete cacao van Houten reso con Buvez du cacao Van Houten!, la cui pubblicazione in italiano, ancora presso Einaudi, dovrà attendere il 2010; due anni dopo Nathalie Baver

4 Secondo Shread i due piani non si possono distinguere sempre con chiarezza: daremo ragione dei rapporti tra i due aspetti, senza pretendere di giungere a una teoria generale. Su temi analoghi cfr. von Flotow 2010, p. 129.

$5 \quad$ Cfr. $\quad$ CV dell'autrice (http://www.analix-forever.com/cgi-

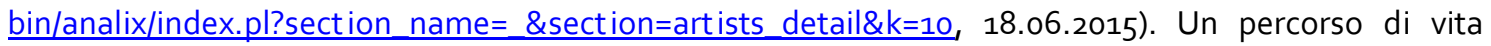
tanto movimentato autorizza l'utilizzo del concetto ampio di traduzione proposto da BachmannMedick, 2009. Processo analogo al percorso traduttivo dei testi di Vorpsi è osservato da Mayanja nella letteratura africana di espressione francese o inglese tradotta in altra lingua europea, che parla di "terzo testo" riferendosi al contenuto in madrelingua che fa da sfondo al testo scritto nella lingua europea e che nel transito a altra lingua europea andrebbe tenuto presente per quanto non recuperabile. (Mayanja, 1999, pp. 26-31 e 46-55). Questa situazione può conoscere tensioni analoghe a quelle descritte da Spivak (2000) a proposito della resa dell'inglese del creolo, della figlia della schiava e dell'abitante dei Caraibi; tuttavia si deve tener presente che per Vorpsi non disponiamo del retro-testo albanese e che la traduzione linguistica avviene tra due lingue di culture un tempo colonizzatrici.

6 Tutti pubblicati presso Einaudi eccetto Vetri rosa: di questi titoli si da ragione più dettagliata oltre.

7 Gallimard, 2014, Viaggio intorno alla madre, trad. di G. Bompiani e B. Torrani. Roma: Nottetempo, 2015 .

8 Per una definizione di auto-traduzione simultanea e differita si veda Grutman, 2008, p. 259. 
rende con Vert venin ciò che in italiano, ancora nel catalogo della casa dello struzzo, appare come La mano che non mordi, e infine Yaun Apperry nel 2007 con Tessons roses mette a disposizione del pubblico francese Vetri rosa apparso in Italia presso Nottetempo. Le traduttrici sono studiose di provata esperienza e competenza nella traduzione dall'italiano, mentre il traduttore si presentava più come artista con familiarità con la performance ${ }^{9}$. II fatto che si tratti prevalentemente di donne che traducono un testo scritto da una donna o ne sostengono l'auto-traduzione è rilevante. Palusci afferma che secondo Nicole Brossard "il linguaggio delle donne rimane fluido, sempre suscettibile di ritocchi e variazioni, esattamente come avviene nel processo traduttivo. Possiamo prendere questo punto di vista come una conferma che la traduzione è (...) un'arte ma è anche un'esigenza femminile" ${ }^{10}$ Pur nella problematicità dell'essenzialismo che fa capolino in questa affermazione e del pericoloso eco della donna come essere da relegare nel settore gerarchicamente minore della traduzione, l'iter della pubblicazione dei testi di Vorpsi pare adatto a verificare il valore e l'entità di oscillazioni tra la formazione di due versioni di un medesimo testo prodotte almeno in parte dall'autrice stessa ${ }^{11}$. Insisto su oscillazione, fenomeno che qui ritornerà più volte, perché in generale con il decostruzionismo e la sua declinazione femminista in ambito di teoria della traduzione la questione dell'autenticità e dell'equivalenza del testo source e del testo target ha assunto caratteri sfumati: declinando in termini semiotici questo stato di oscillazione

9 Véron è traduttrice dall'inglese e dall'italiano, Baver solo dall'italiano ed alla traduzione affianca scrittura letteraria in proprio, Pozzoli, responsabile della sezione italiana di Actes Sud, ha tradotto S. Benni, M. Maggiani, A. M. Ortese e V. Magrini; Apperry, romanziere e autore teatrale, all'epoca della pubblicazione di Vetri rosa non aveva al suo attivo traduzioni in italiano. Possiamo valutare il significato della presenza di traduttrici, se ricordiamo casi di ciò che in appoggio a recenti elaborazioni di J. Butler potremmo definire desoggettivazione come quelli di Clara Schumann e più recentemente di Susanna Brenner-Rademacher (cfr. Chamberlain 2000 e Snell-Hornby 2001, pp. 23-24). Anche risalendo a anni più prossimi alla pubblicazione dei testi di Vorpsi, nonostante gli importanti lavori di von Flotow e Spivak, Stolze ${ }^{3} 2001$ non offre sezioni dedicate alla riflessione femminista sulla traduzione. II fatto non può che stupire se si pensa che Amati Mehler, Argentieri, Canestri 1990 dal significativo titolo La babele dell'inconscio ha in "Ident ità e ident ità di genere attraverso le lingue" un capitolo sulla questione di genere nella traduzione psicanalitica (Id., pp. 87-128).

10 Palusci, 2010, p. XVI.

11 Se Râbacov 2013, p. 68, tra le cause dell'auto-traduzione menziona, sul piano individuale, sfiducia e perfetto bilinguismo e, sul piano sociale, società multiculturale, domino culturale e carattere elitario di una lingua, in Vorpsi, in difetto di dati, si può supporre un trilinguismo (albanese, italiano e francese) che per mot ivi legati a dominio culturale ha portato l'autrice a scegliere una delle lingue di prestigio da lei padroneggiate per esprimere contenuti che provengono dalla cultura della madrelingua minoritaria che quindi risulta tradotta nelle altre due. Sulla traduzione come riscrittura e sulla cultura come testo soggetto a cont inue traduzioni cfr. rispettivamente Borutti Heidemann 2012, pp. 163-185 e BachmannMedick 2009. Più specificamente ma con ampio respiro storico sull'auto-traduzione cfr. Hokenson Munson 2007 e Forster 1970; Santoyo 2005 offre una rassegna storica in difesa della consistenza dell'auto-traduzione, della quale condividiamo la preoccupazione di rivalutare l'importanza del fenomeno auto-traduttivo, che tuttavia richiederebbe un rapporto ponderato e qualitativo tra traduzione, creazione e contesto. 
intertestuale, diciamo che cercheremo di verificare le oscillazioni di significato che si possono registrare nella intentio operis di due versioni di superficie della medesima materia $^{12}$.

Ritorniamo brevemente alle vicende editoriali dei testi di Vorpsi: i percorsi più atipici sono toccati in sorte al racconto lungo Vetri rosa e al romanzo breve Il paese dove non si muore mai, che si scostano dalla norma, I'uno, per le forme editoriali nelle quali si è materializzato e, l'altro, per la procedura di traduzione. Il primo testo è apparso anzitutto come oggetto d'arte per bibliofili costituito da una cassetta in cui sono adagiati il fascicolo contenente il testo e lamine fotografiche che rielaborano un soggetto pittorico tratto da Lorrain. L'oggetto è frutto della collaborazione di tre artisti gravitanti attorno a gallerie d'arte attive in Svizzera: Vorpsi si è occupata del testo letterario presente in versione trilingue, M. Collishaw ha fornito le immagini delle lamine e P. Cramer ha disegnato il contenitore. In seguito, presso Actes Sud, è apparsa la traduzione francese, col titolo Tessons roses, che rinvia in maniera più chiara rispetto al titolo italiano al motivo del racconto, sia attraverso il valore primario di tesson, che indica oggetti infranti mentre l'italiano vetri solo per metonimia e per I'uso del plurale indica vetri infranti, sia per l'immagine di copertina, che, costituita da una composizione della stessa Vorpsi, ritrae un viso scorciato, presumibilmente di donna, colto nell'atto di mordere una collana di perle rosate; l'edizione francese e quella italiana differiscono iconograficamente anche all'interno in quanto mentre quella francese presenta immagini intercalate al testo, in quella italiana immagini solo in parte coincidenti con quelle della versione francese sono raccolte in coda al racconto. Nelle diverse forme tipografiche assunte da Vetri rosa si può riconoscere ciò che A. Berman designa come traslazione, vale a dire trasformazione di un testo che investe tanto gli aspetti linguistici sostanziali quanto quelli solitamente considerati accessori, come le soglie ${ }^{13}$.

Il paese dove non si muore mai, apparso in francese col titolo Le pays où l'on ne meurt jamais, stando alle notizie di copertina, sarebbe stato tradotto con la collaborazione dell'autrice: si tratterebbe quindi di un caso di auto-traduzione simultanea sostenuta da madrelingua o di traduzione sorvegliata e certificata, secondo il peso che vogliamo dare al contributo delle due autrici ${ }^{14}$. Anche questo

12 Per una breve trattazione del rapporto tra traduzione, equivalenza, traccia e critica femminista cfr. Furster Ortuño, 2008; per la collocazione del ruolo del traduttore e della traduttrice si veda Borek 1998 e per un'esemplificazione delle competenze linguistiche dei soggetti coinvolti basata sulla traduzione in francese di Berliner Kindheit di Benjamin Kappeler, 2008, pp. 28-62.

13 L'oggetto d'arte contiene la traduzione in francese e in inglese del testo italiano.

14 Non disponendo di dati cronologici sulla relazione tra i due testi, parleremo di versioni senza distinzione tra originale e copia: ci concentreremo sull'analisi comparata del significato dei due testi secondo un'analisi minuziosa dei fenomeni linguistici che ci parranno più interessanti. R. Grutman, in Auto-translation (Baker, 1998, p. 19) sost iene che l'autotraduzione è più un processo di doppia scrittura che di doppia attività e quindi "(C)ome risultato, la precedenza dell'originale non è affatto una questione di "status and standing", di autorità, ma diventa „puramente temporale". La distinzione tra 
romanzo, come Vetri rosa, ha visto l'utilizzo di materiale fotografico dell'autrice: tuttavia la struttura semiotica de Il paese dove non si muore mai risulta più semplice in quanto l'unica immagine presente si trova sulla copertina e le due edizioni differiscono solo per il taglio e per la tonalità cromatica. Il soggetto ritratto è una figura femminile di età compresa tra la tarda adolescenza e la maturità; in piedi, indossa un abito rosso a spalline sottilissime e gonna ampia con pieghe verticali. La donna si trova in un ambiente che, per il poco che di esso si vede - solo una parete e parte del tetto -, appare spoglio; prende luce da un lucernario di cui l'obiett ivo cattura solo uno scorcio nell'angolo superiore destro dell'immagine. Il lucernario e l'ambiente sono in contrapposizione cromatica in quanto questo è caratterizzato da tonalità di rosso sangue, mentre quello è di color azzurro; la struttura della presa di luce getta sulla donna un'ampia ombra che oscura completamente il capo e la spalla sinistra, mentre una linea più sfumata attraversa il corpo a partire dal gomito destro per spegnersi all'altezza del bacino. Per quanto si può vedere dello spazio, pare che la donna si trovi con la fronte lievemente inclinata e appoggiata a una parete. La soglia estrema del testo, per quanto riguarda le possibili connotazioni cromatiche, sembra quindi evocare tanto passione quanto violenza di atti cruenti, mentre, per la postura della donna, traducendo in linguaggio figurato il contenuto dell'immagine, si è portat $i$ a pensare a situazioni nelle quali il soggetto femminile è messo alle strette e deve abbassare la testa. Tra le due realizzazioni grafiche notiamo che quella francese è caratterizzata da un viraggio cromatico verso il mattone nella tonalità che domina la donna e lo spazio chiuso, cosicché l'associazione con atti cruenti risulta o attenuata, se si è inclini a pensare a una parete di mattoni in argilla, o modificata, se si pensa al colore del sangue rappreso. L'edizione francese fa intravedere non solo l'apertura del lucernario, ma anche la finestra che lo chiude; inoltre la stessa realizzazione concede spazio alla donna sino alle ginocchia dando maggior verticalità alla composizione e rilevando maggiormente le ombre che attraversano il corpo della donna. Tra le due immagini, pur nell'unità temat ica di fondo, not iamo quindi una leggera variazione che nella versione per il pubblico italiano pare accentuare la tensione drammatica.

A un confronto delle diverse versioni di tutte le opere di Vorpsi è risultato che il testo con i maggiori scarti di formazione tra le due versioni è proprio quello tradotto in collaborazione: si tratta di una possibilità insita nell'auto-traduzione o nella

originale e (auto-)traduzione collassa, lasciando spazio a una terminologia più flessibile nella quale i due testi sono considerati come „varianti" o „versioni" di pari stato, "auto-traduzioni simultanee". Nello stesso volume si veda Pseudotranslation di D. Robinson, (pp. 183-185).

Non disponendo di dati cronologici sulla relazione tra i due testi, parleremo di versioni senza distinzione tra originale e copia: ci concentreremo sull'analisi comparata del significato dei due testi secondo un'analisi minuziosa dei fenomeni linguistici che ci parranno più interessanti. R. Grutman, in Auto-translation (Baker, 1998, p. 19) sostiene che l'autotraduzione è più un processo di doppia scrittura che di doppia attività e quindi "(C)ome risultato, la precedenza dell'originale non è affatto una questione di "status and standing", di autorità, ma diventa „puramente temporale". La distinzione tra originale e (auto-)traduzione collassa, lasciando spazio a una terminologia più flessibile nella quale i due testi sono considerati come „varianti" o „versioni" di pari stato, "auto-traduzioni simultanee”. Nello stesso volume si veda Pseudotranslation di D. Robinson, (pp. 183-185). 
traduzione in collaborazione con l'autrice del testo. Infatti, secondo le convenzioni editoriali moderne e le aspettative prevalenti del mercato, il traduttore o la traduttrice che lavori in autonomia deve rendere nella lingua target il testo della lingua source secondo una più o meno problematizzata e manifesta fedeltà all'originale; più liberamente, l'auto-traduzione assistita può produrre un altro testo frutto del dialogo tra autore o autrice e traduttore o traduttrice e della riflessione di poetica e stilistica cosi indotta. Non dobbiamo dimenticare che i testi che leggiamo sono passati al vaglio del lettorato del cui intervento sul manoscritto non abbiamo tracce e nemmeno sappiamo se le procedure presso le due case editrici siano avvenute parallelamente o se la versione italiana abbia fatto tesoro delle revisioni richieste dall'editore francese, cosicché il punto d'enunciazione risulta sfuocato ${ }^{15}$. Riassumendo quanto anticipato, nelle pagine seguenti cercheremo di valutare gli effetti della procedura auto-traduttiva messa in atto da soggetti femminili sui due prodotti di superficie: viste la tematizzazione dei rapporti di genere e l'ambientazione in contesto fortemente patriarcale, valuteremo se nelle due versioni della medesima vicenda si possano riscontrare differenze denotative e connotative in relazione a oggetti, in parte tradizionalmente ascritti all'area di pertinenza femminile; in questa ipotesi di lettura, dopo collazione dei passi devianti, abbiamo scelto brani in cui i punti di frizione tra i due testi emergono direttamente o indirettamente in relazione alla rappresentazione degli oggetti anche perché, secondo una concezione ingenua del linguaggio, dovrebbero essere gli elementi referenziali meno ambigui e quindi meno sensibili a contrattazioni in ambito traduttivo ${ }^{16}$; del materiale presentiamo prima le soglie dei due testi e le devianze del capitolo iniziale; poi raccogliamo il materiale per ambiti semantici che ci sono sembrati rilevant $i$; concludiamo con un'osservazione di portata generale sul rapporto tra auto-traduzione e critica letteraria.

Si è già ricordato che l'autrice del testo di partenza è di madrelingua albanese; se si aggiunge che dichiaratamente considera l'italiano come sua lingua di accoglienza, si deve supporre che, a livello tematico e linguistico, nell'elaborazione della storia ci sia stato l'albanese come lingua quotidiana di scambio e come lingua di espressione culturale, mentre si può pensare che l'italiano abbia rappresentato la lingua straniera in cui l'autrice si sentiva più a suo agio per motivi esistenziali e per motivi editoriali ${ }^{17}$.

15 A tale proposito si potrebbe mettere in valore la concezione di enunciatore e enunciatario come presenze spettrali dell'enunciazione messa a punto da Courtés, 1991, p. 255.

16 Simon 1996, p. 30 contrappone friction a affinity creando due poli ai cui estremi collocare i due potenziali atteggiamenti simpathetic e antagonistic verso il testo da tradurre. La costellazione dei quattro concetti non può esser applicata nell'ambito dell'auto-traduzione assistita. La frizione è, nella nostra prospettiva, il contrasto tra due versioni di superficie in due lingue diverse della medesima materia narrativa.

17 Cfr. intervista 4.10.2007 in http://www.lanotadeltraduttore.it/intervista_ornela_vorpsi_scrittrice.htm (28.05.2016). Sulle variabili che condizionano il processo di produzione e pubblicazione di una traduzione si veda Munday, 2001, pp. 126-160. Sul rapporto tra luogo di enunciazione della traduzione e 
Su questo sfondo si deve supporre che, nel momento in cui l'autrice si trovava in Francia, sia avvenuta la contrattazione tra lei, autrice della versione italiana e cotraduttrice in francese, e la traduttrice di madrelingua francese ${ }^{18}$.

Fermo restando lo spazio d'indeterminatezza creato dall'inaccessibilità ai manoscritti consegnati agli editori, si può lavorare con l'ipotesi che i passi dei testi pubblicati che presentano maggiori differenze nella formazione possono esser considerati come tracce di una probabile rielaborazione più tormentata e potrebbero essere quelli che hanno impegnato maggiormente i due soggetti coinvolti nella traduzione di strutture profonde nei testi di superficie delle due lingue straniere, senza diment icare due ulteriori obiezioni di fondo: anzitutto, da un lato, può darsi che discussioni su termini e passi non abbiano lasciato tracce riconoscibili al confronto dei testi in francese e in italiano, mentre, da un altro lato, può darsi che brani che appaiono precipitato di accesi dibattiti in realtà siano il frutto di soluzioni di comodo non problematizzate dalle traduttrici. Si consideri anche che una completa presentazione dei meccanismi di traduzione e del loro significato per la forma del testo di arrivo richiederebbe un'analisi ponderale che valutasse il peso dei singoli fenomeni di acclimatamento nella totalità del testo ${ }^{19}$. Infine ripetiamo che dietro I'italiano e il francese c'è senza dubbio l'esperienza vissuta dell'albanese e in albanese da parte dell'autrice.

Prima di esplicitare in forma concisa le domande con le quali interrogheremo le due versioni del romanzo breve, osserviamo che il testo che analizzeremo, sul piano della distribuzione della materia informativa, è cost ituito da frammenti narrativi con deboli legami spazio-temporali e che gli agenti in essi presenti non sono sempre gli

ricezione della stessa si veda Arrojo 1994, pp. 39-44, in cui l'autrice insiste sul ruolo della comunità interpretativa.

Cfr. intervista

4.10.2007

in

http://www.lanotadeltraduttore.it/intervista_ornela_vorpsi_scrittrice.htm (28.05.2016). Sulle variabili che condizionano il processo di produzione e pubblicazione di una traduzione si veda Munday, 2001, pp. 126-16o. Sul rapporto tra luogo di enunciazione della traduzione e ricezione della stessa si veda Arrojo 1994, pp. 39-44, in cui l'autrice insiste sul ruolo della comunità interpretativa.

18 Trattando la presenza di più versioni del medesimo testo nella produzione di V. Flusser, Guldin afferma che „un'altra funzione dell'ult ima versione è accumulare tant i punt i di vista quanto è possibile sullo stesso oggetto: il pensiero originale. In questo modo la pluralità delle differenti lingue trova la sua via nella complessa unità di soggetto". Guldin, 2004, p. 113. Nella stessa direzione si muove la distinzione tra simultaneous auto translation e delayed auto-translation proposta da Grutman, op. cit., p. 20: la differenza tra i due processi consisterebbe nel fatto che nel primo la traduzione avverrebbe durante la stesura dell'opera. Nel caso dei testi di Vorpsi possiamo pensare a versioni che crescono da un'unica materia tematica, o di un "dinamico processo di comprensione e re-espressione di idee". Cfr. M. Salama-Carr, Interpretive approach, in Baker, 1998, p.113.

19 Nella piega di questo dato quant itativo si nasconde la problematicità della definizione dell'unità di traduzione: si traduce una parola, una frase, un capoverso o un testo intero? Ciò che a livello di frase può apparire eccessiva libertà, può risultare più efficace, se si considera un segmento più ampio. La tensione tra informativo e formativo è alta: la sua valutazione richiede una cautela di cui è traccia il nostro procedere per analisi minuziose, che, se hanno richiesto riflessione allo scrivente, richiedono pazienza al lettore e alla lettrice. 
stessi, cosicché, più che da un soggetto costantemente presente sulla scena narrativa, la cont inuità è assicurata dalla temat ica, vale a dire da rapport i tra uomini e donne caratterizzati da forti tensioni su uno sfondo costituito da un'Albania che risente degli effetti della dittatura comunista, ma che continua ad esser dominata da strutture patriarcali. L'immagine dell'Albania è decisamente sfuocata per quel che riguarda le determinazioni di tempo e di luogo, cosicché la narrazione acquisisce valore tendenzialmente universale.

Dato il quadro tematico delineato, porremo le due seguent i domande: si possono riconoscere specifiche sensibilità rispetto alla questione di genere con particolare riferimento al ruolo degli oggetti nella costruzione delle due versioni del testo letterario Il paese dove non si muore mai / Le pays où l'on ne meurt jamais? In caso affermativo, si possono individuare nelle differenze tra i due testi ambiguità che facciano pensare a scarsa trasparenza proprio in relazione a questioni di genere veicolate da oggetti e quindi possano portare a giudicare come et icamente confusa l'operazione auto-traduttiva ${ }^{20}$ ? Sono quesiti circoscritti adatti a una lettura a ridosso del testo e agli spazi di un contributo per rivista ${ }^{21}$.

\section{Oscillazioni e ambigui slittamenti}

Prendiamo l'avvio dalle soglie linguist iche dei due test $i$. Rispetto ad altre opere di Vorpsi, le due versioni di quella sull'eternità dell'albanese presentano titoli con un forte grado di corrispondenza. Se aprendo i due libri, l'indice dei capitoli ci rivela solo due lacune che consistono nella mancanza del titolo complessivo dell'opera nell'edizione francese e nella cassazione di un corrispondente di Terra promessa nell'Epilogue, scorrendo i titoli dei capitoli invece ci imbattiamo in uno slittamento non indifferente: "Macchie" e „Tuorli d'uovo" guadagnano due posizioni rispetto ai corrispondenti „Taches” e "Jaune d'oeuf" dell'edizione francese. L'inversione non è priva di significato perché la versione francese crea un rapporto di contiguità tra la

20 L'etica della traduzione rimanda a Hirsch 1997 che sviluppa tematiche da W. Benjamin e J. Derrida ma soprattutto, da E. Levinas. I concetti messi in campo dall'autore sono libertà, fedeltà, differenza, alterità, responsabilità, ai quali, volendo declinare l'approccio secondo le particolarità dell'autotraduzione, si dovrebbero aggiungere almeno, onestà, costruzione di sé e auto-trasparenza. Per una più articolata valutazione dei rapporti tra etica e traduzione, cfr. Babel, 2015. L'autore nell'introduzione mette in rilievo come l'aggettivo redlich traducibile con onesto ha più a che fare con il discorso ben costruito, con il ben parlato (gut geredet), che con la Ehrlichkeit cosicché, aggiungiamo noi, il discorso ben formato si colora di qualità et iche (Id., p. 11). Sul nesso tra et ica e traduzione nell'ult imo Berman in prospett iva femminista si rimanda a Simon, 1996, pp. 25-38.

21 Hokeson Munson (2007, p. 2) per un testo in auto-traduzione propongono domande più generali: i due testi sono entrambi originali? Sono entrambi completi? L'auto-traduzione è un genere a sé? Le due versioni appartengono solo a una lingua o storia letteraria? Quali sono le possibilità di commensurabilità delle due versioni? Attraverso le nostre analisi cercheremo di giungere a conclusioni, che siano interessanti anche se non di respiro tanto ampio quanto quelle cui potrebbero portare le domande or ora menzionate. 
Corona di Cristo, che a una prima lettura può ben esser la corona di spine, e le macchie, che, dopo la possibile allusione alla passione di Cristo, sono fortemente attratte o nell'area figurata del peccato come atto che intacca l'immacolato stato della coscienza innocente o nel dato concreto del sangue versato da Cristo. In questa prospettiva la mancanza del titolo generale del testo e quello dell'epilogo possono acquistare maggior peso rispetto a quanto sospettato: viene tolto nella versione francese un immediato riferimento alla sfera della redenzione e della vita eterna. Ecco come suona la serie nella versione italiana: „Campa, campa e non crepa l'albanese”, "Macchie”, „Tuorli d'uovo", "Corona di Cristo”, "Albania sensuale”, „Bel-Ami”, "Sogno”, „Storie di donne”, „Acque”, „Arance di Tirana”, "Giardino d'infanzia”, „I dervish delle meraviglie", "Sangue rosso sulla neve bianca", "Il coro a due voci", "Epilogo: Terra promessa". La serie di titoli si apre con una terra (il paese del titolo complessivo) e si chiude con un luogo di redenzione, secondo una possibile prospettiva biblica, anche se, dalla semplice lettura dell'indice, non è chiaro se il paese del titolo, quello in cui non si muore mai, sia la terra promessa o altro luogo. I capitoli attraverso i quali avviene il transito sono costellati di oggetti: alle già citate macchie, che, se intese in senso letterale, presuppongono un supporto materiale, seguono sostanze dal potenziale uso alimentare; immediatamente dopo troviamo tuorli d'vovo, di cui non si conosce la destinazione d'uso, che potrebbe esser cosmetica; infine, con un salto in avanti, ci imbattiamo in acque e arance. Da notare l'ambiguità connotativa del giallo non menzionato ma evocato da arance e tuorli. Analizziamo anzitutto le potenziali catene associative dei due oggetti; da un lato la tonalità cromatica è associata a un frutto il cui nome è usato per indicarne una varietà intensa, l'arancione, che etimologicamente deriva da auratum cioè dorato; dall'altro viene evocata dal tuorlo associato nella lingua colloquiale al giallo d'vovo e alla chiara. La connotazione del frutto, con forte coesione semantica rimanda al sole e per la forma e per le latitudini calde in cui cresce l'albero, mentre quella del tuorlo appare meno coesa perché rimanda, da un lato, alla vita allo stato embrionale, e dall'altro, per metonimia, al colore lattiginoso, cioè chiaro, dell'albume. "Corona di Cristo" e "Giardino d'infanzia", con uno slittamento verso il Giard ino terrestre o verso l'orto del Getsemani, luogo del tradimento di Cristo da parte di Giuda, collocano nuovamente I"'Albania sensuale" e il "Sangue rosso sulla neve bianca" in una prospettiva cristologica, che potrebbe trovare ambientazione in una chiesa con "Il coro a due voci" o in un rituale esotico o esoterico orchestrato da un "Dervish delle meraviglie”. „BelAmi" e "Sogno" sembrano esser extravaganti e lasciar spazio alle più diverse associazioni mentali. Leggendo il testo, ci si renderà conto che la corona di Cristo è la passiflora, il che fornirà ulteriore pezza d'appoggio per la lettura cristologica; si avvicinerà la corona o al sangue rosso sulla neve di cui si narra nel testo, visto che molte varietà della passiflora hanno fiori rossi, oppure agli organi sessuali del medesimo fiore che ricordano la figura della corona di spine insanguinate. 
Veniamo ora alla traduzione dei titoli dei capitoli. Il titolo del primo presenta differenze sostanziali nelle due versioni. A un "Il ne rompt pas l'Albanais, mais il plie tant qu'on voudra" $(9)^{22}$ che fa pensare a qualcosa di infrangibile anche se piegabile a piacimento, con inversione dell'ordine e del valore secondo i quali ci si aspetterebbe la collocazione dei due enunciati ${ }^{23}$, risponde un ben più forte "Campa, campa e non crepa l'albanese" (5). Il titolo francese ha una certa musicalità dovuta all'insistenza sulla vocale aperta a modulata attorno a poche vocali di diversa apertura e alla chiusura in a che richiama la particella di negazione pas. A livello di contenuto il titolo rievoca il frammento di Pascal (Pensées, VI, 347, ed. Br.) dell'uomo come canna pensante: „L'homme n'est qu'un roseau, le plus faible de la nature; mais c'est un roseau pensant. II ne faut pas que l'univers entier s'arme pour l'écraser: une vapeur, une goutte d'eau, suffit pour le tuer. Mais quand l'univers l'écraserait, l'homme serait encore plus noble que ce qui le tue, puisqu'il sait qu'il meurt, et l'avantage que l'univers a sur lui; l'univers n'en sait rien. Toute notre dignité consiste donc en la pensée." La struttura del giro di frasi pascaliano conferma l'impressione che sia avvenuta un'inversione del rapporto tra i contenuti: infatti nel testo del filosofo l'avversativa introduce il tema della rottura o dell'annientamento, mentre nel titolo dell'opera di Vorpsi introduce la resistenza dell'albanese pur nella sua cedevolezza. L'inversione potrebbe marcare con maggior forza le sofferenze cui è sottoposto l'albanese a causa dei continui piegamenti: il rilievo che solitamente si è portati ad attribuire alle avversative per il loro valore corrett ivo rispetto all'enunciato cui sono legate andrebbe in questa direzione. Campa richiama una serie di espressioni idiomatiche di diversa coloritura tutte afferenti alla sfera di una vita dura: campare di qualcosa, campare di miseria, tirare a campare, fino ai proverbiali campa cavallo che l'erba cresce e chi campa tutto l'anno vede tutte le feste. Crepa come verbo col significato di morire è di registro basso e viene solitamente usato in contest i affetti da pena, miseria e violenza; come sostantivo invece indica una forte frattura procurata in un solido, come una roccia o un oggetto in terracotta. Col titolo del capitolo di apertura le due versioni mettono in diverso rilievo il tema della compromissione del funzionamento dell'animale umano di nazionalità albanese che a livello fisiologico ha il suo corrispettivo nella morte.

Riassumendo le osservazioni sui due indici, possiamo concludere che nel complesso le due versioni, con strategie leggermente diverse, collocano la narrazione in un mondo precario popolato di beni elementari - acqua, vova, frutta - present i in un giardino - non in un orto-: i frutti unitamente al sangue sono caratterizzati da qualità cromat iche collocabili nell'area di tonalità calde tra giallo e rosso, e proiettat i

22 Risultando chiaro dalla lingua la versione dalla quale si cita, tra parentesi si riporta il solo numero di pagina.

23 Ci si aspetta prima l'enunciato che manifesta il tentativo di rottura e poi quello che ne denuncia la vanità. 
in una prospett iva di redenzione che tuttavia, nello sviluppo della vita menzionata nel suo inizio embrionale, appare costellata di incidenti legat $i$ a dolore e passione.

Passiamo ora al testo. Sfogliando parallelamente le prime pagine, si nota la diversa distribuzione degli spazi bianchi: la versione francese appare più frammentata rispetto a quella italiana; proseguendo nella lettura a volo, dobbiamo tuttavia constatare una rarefazione delle differenze nelle spaziature. A livello qualitativo il testo francese sembra contraddire la tendenza all'uso espressivo della spaziatura; infatti, se nella versione italiana dopo solo tre pagine leggiamo in posizione rilevata il motivo centrale del racconto („Ecco come muoiono gli altri”, 7), quella francese lo presenta in un lungo capoverso e con perdita di eminenza (,Voila comment les autres meurent", 12), anche se a compensazione di ciò il capoverso precedente ha una porzione esplicativa che manca a quello italiano: a "C'est la vie ... II est rentré du travail, il a mangé et il est mort" risponde un laconico „La vita è imprevedibile!"

In apertura di racconto si trova un'ipotetica spiegazione dell'origine del coraggio da attribuire agli albanesi che mette in campo uno strano oggetto: De là, aussi, l'absence de peur - à moins qu'elle ne soit due à la forme de poterie mal façonnée qui est celle du crâne des autochtones, tordu et aplati, royale demeure de l'unsouciance, sinon de l'inconscience. ${ }^{24}$ (10), che nella versione italiana suona come segue:Da ciò anche l'assenza di paura - a meno che questa non sia dovuta alla forma del cranio storto e piatto, dimora regale dell'insofferenza, se non dell'incoscienza. (5) La differenza più rilevante tra le due versioni è la mancanza nel testo italiano della metafora del mal formato recipiente in terraglia per il cranio del tipico albanese; mancanza tanto più gravida di conseguenze se si osserva che il veicolo del senso, è anteposto rispetto all'oggetto creando cosi una certa tensione nel lettore che mentre legge dell'articolo di vasellame si chiede a cosa si riferisca. Inoltre il cranio nella versione albanese è qualificato come appartenente agli autochtones, termine che contribuisce ad elevare il registro della versione francese, oltre ad aggiungervi un'informazione di lieve patina scientifica. Il testo francese sembra suggerire corrispondenza tra fisiologia e psicologia e quindi una naturalizzazione dei comportament i degli esseri umani.

Ritornando alle spaziature e allo spazio concesso alle parti esplicative, si può sostenere che la strategia di composizione testuale sembra esser guidata in modo da creare, almeno inizialmente, una versione francese frammentata e una italiana più compatta. Nel medesimo capoverso sulla morte, la facilità dei rapport i sessuali, l'altra questione centrale del libro, è isolata a capoverso nella versione italiana, mentre è inserita a fine periodo, dopo due punti, con valore esplicativo nella versione francese. Ecco i due testi: Una di queste senza esagerare è quasi il centro della loro vita. La

24 Se unsouciance sia neologismo o refuso non si può dire, comunque, reso in italiano, corrisponderebbe più a trascuratezza che a insofferenza e, volendo tentare un neologismo, si potrebbe proporre incuratezza. 
questione della puttaneria. (7); L'un d'entre elles, et je m'exagère nullement, est le centre de leur vie: la question de la putinerie. (12)

Abbandonando la questione della forma testuale e proseguendo nell'area tematica di nostro interesse, l'italiano ha un'eufemistica designazione dei genitali esterni femminili con un generico "là sotto" (7), che manca in francese, lasciando incertezza su ciò che verrebbe ricucito: l'espressione italiana sembra riprodurre una reticenza da attribuire a una sorta di discorso indiretto libero di registro popolare in armonia con la soppressione dell'autochtone di cui abbiamo dato conto.

Cont inuando nella lettura del capitolo, notiamo che "Dans la rue, leurs regards te pénètrent jusqu'à la moelle des os, si profondément que ton être devient transparent. Une fois en toi, leur fouille est méticuleuse." (10) nella versione italiana slitta di un paio di pagine senza manifest i mot ivi e così suona; "Quando passi per la strada, i loro sguardi t'incrociano penetrandoti fino al midollo, cosi a fondo che il tuo essere diventa trasparente. Una volta dentro di te, questo sbirciare diventa un'arte meticolosa."(8) L'aggiunta d'incrociare, che significa percorrere metodicamente una zona di mare o di cielo in un'azione esplorativa o di guerra, sembra rinviare a un rapporto non cameratesco tra i due sessi e può esser compensato dal francese da fouille che, pur usato metaforicamente, indica un atto meno neutro rispetto allo sbirciare perché implica l'ingerenza nel corpo altrui.

I genitali maschili, mistero nascosto nei pantaloni degli uomini secondo l'istanza narrante, sono introdotti nel testo francese secondo la modalità della probabilità („semble-t-il", 14) mentre nel testo italiano si manifesta l'incertezza delle relazioni tra di essi e la puttaneria ("ha qualcosa a che fare con", 8). II tremito della voce che enuncia la previsione del futuro professionale della giovane protagonista e proveniente presumibilmente dall'ambito familiare, in francese acquista la qualifica intenzionale di moqueur (15), che in italiano può esser implicata nelle particelle interiettive "eh... eh..." (9).

La previsione di una gravidanza indesiderata della giovane protagonista è introdotta in entrambi i test $i$ al futuro e con simile registro linguistico: „tu ne vas pas tarder à devenir une belle salope" (15) appare più analitico rispetto a "diventerai una gran troia” (9), ma poi la versione italiana marca con forza che „non si può fare niente, mica l'abbiamo scelta noi, una come te! Mangeremo la vergogna col pane, ecco cosa ci resta da fare! Un giorno ci piomberai a casa col ventre riempito!", mentre il testo francese con l'uso del presente "nous devons manger notre honte avec le pain" rende drammaticamente attuale la vergogna, per andar al futuro solo in seguito (on $n^{\prime} y$ pourra rien). Pare che, pur con mezzi diversi, i due testi dal punto enunciativo dell'anonimo gruppo dei familiari inducano l'idea di una certa inevitabilità dell'istinto sessuale e delle sue conseguenze, confermato da una concezione passiva dell'atto sessuale della donna. L'effetto della fecondazione viene reso con uno stato in francese (un gros ventre) mentre in italiano è realizzato con la forma passiva contratta nel participio passato in funzione attributiva (ventre riempito), che tendenzialmente evoca 
l'immagine di qualcosa che rimane passivo (la donna evocata metonimicamente) e di qualcuno o qualcosa (l'uomo complemento d'agente non espresso) che riempie: il corpo della donna risulta cosi oggettivato e sottoposto a una violenza che sarebbe stata attenuata con l'aggettivo pieno, più vicino alla forma francese. Una tendenza a slittamento verso registri più bassi nell'italiano viene confermata da "chiavata" $\mathrm{e}$ "scopata" (10), che corrispondono nel testo francese a "fornication" e "accouplements", anche se il capoverso, chiudendo con una allegra „partie de jambes en l'air" (16) recupera in parte il tono popolaresco, traducendolo in chiave comica e dinamica. A fine capitolo si parla di marmellata concessa per compassione alla ventura prostituta durante una malattia; l'oggetto nella versione francese, dopo che la convalescente ha raggiunto la guarigione, si allontana „en un tournemain” (17), che al processo di probabile ritorno alle consuete tensioni familiari dà un'accelerazione assente nell'italiano.

Pur ponendoci come filo rosso per la nostra indagine gli oggetti, non possiamo non notare che nel primo capitolo or ora passato al setaccio, ci viene presentato I'unico rilevante cambiamento nella struttura dei personaggi tra le due versioni: chi rimprovera la protagonista per il suo destino di donna di malaffare nella versione francese sono la madre e la nonna (15), mentre in quella italiana diventano la zia e una cugina (9), vale a dire non persone direttamente coinvolte nel processo educativo.

Abbandoniamo l'analisi progressiva pagina per pagina per anticipare un'impressione generale da verificare per campionature tematiche. La versione francese sembra caratterizzata da maggior dinamicità, che si può esser portati ad attribuire alla maggior segmentazione del testo sia a livello tipografico sia a livello sintattico. Spazi bianchi, frasi brevi e frequente concessione della parola ai personaggi sembrano dare maggior agilità alla narrazione e alle descrizioni. Per quanto riguarda il registro linguistico, il testo francese sembra preferire forme non volgari e termini denotat ivamente precisi. Inoltre si deve rilevare oscillazione nell'uso dei tempi verbali tra passato, presente e futuro. Sul piano tematico il testo soggiacente alle due versioni sembra invitare a pensare a una redenzione impossibile per la donna segnata dal marchio di una sessualità incontrollabile e infamante. Ma vediamo nel dettaglio se e come vengono variati alcuni contenuti che ordineremo secondo nuclei tematici affini.

Uno dei fili rossi della vicenda è cost ituito dalla fatale vocazione alla „puttaneria”, cui secondo una non meglio qualificata istanza narrante, sarebbero votate le donne avvenenti e in special modo la figura femminile più presente sulla ribalta della serie di frammenti narrativi. La versione italiana con il maiuscoletto in posizione centrale imita il risalto tipografico che si può immaginare in un'insegna di esercizio pubblico (PUTTANERIA E CULO VENDESI, compagnia padre \& figlia):

Quanto insistevano su quella bandiera! „Quando tu alzerai la bandiera, quando voi due alzerete la bandiera ... Non è lontano il giorno che vedremo alzata la bandiera". Io, non so perché, la vedevo rossa, una 
bandiera rossa che sventolava sul portone del negozio: PADRE \& FIGLIA CULO VENDESI. „Per adesso devo aspettare” (41)

La versione francese sceglie un trattamento meno mimetico:

Traînerie et Putinerie en tout genre, vente en gros, compagnie Père \& Fille. Comme ils insistaient sur ce drapeau! „Quand hisseras le drapeau ... Quand vous hisserez ensemble le drapeau. II n'est pas loin le jour où l'on verra hissé ce drapeau." Et moi, je ne sais pas pourquoi, je le voyais rouge ce drapeau, qui flottait au-dessus de la devanture du magasin "Père \& Fille, sexe en vente" "Pour l'instant cependant, je dois prendre mon mal en patience. (57-58)

A livello semantico e in riferimento alla fet icistica presenza di una parte del corpo umano il testo francese è più coerente: se un „culo" si può vendere, non lo si può dire nel medesimo senso di „puttaneria”, perché si può vendere ciò che l'inclinazione alla puttaneria mette a disposizione. Inoltre, mentre la versione italiana ha un lapidario "Per adesso devo aspettare”, quella francese con „Pour l'instant cependant, je dois prendre mon mal en patience” (58) rinforza lo slittamento temporale („pour l'instant” e "cependant") e rende la dimensione dell'attesa con una metonimia. L'espressione della disperazione del padre per il suo destino con un enfatico "Lui, I'homme, pleurait" (54) non trova corrispondenza nella versione italiana: differenza di non poco rilievo visto che rubrica il padre sotto la categoria di soggetto di qualità virili, sment ite per l'allusione alla sua inclinazione al pianto, che, secondo lo stereot ipo coerente con l'immaginario presente nel testo, sarebbe negato ai maschi.

Che la questione delle relazioni sessuali non regolate dal matrimonio sia il centro temat ico della raccolta di framment i narrat ivi è confermato del capitolo Acque che, in un'atmosfera senza tempo, colloca il resoconto della storia di due donne, Blerta e Dorina, che, non sapendo affrontare l'umiliazione della manifestazione della gravidanza extra-matrimoniale, si suicidano. La scarsa funzionalità estetica di Dorina è resa con „problemi di salute alle gambe” che „di tanto in tanto le causano dei cedimenti" anche se "però un po' di sesso si può fare" (6o), mentre il testo francese, con strategia allusiva che sembra riprodurre l'anonima voce della comunità maschile, recita „sexuellement, par contre, elle a ce qu'il faut" nonostante fosse affetta da una deformazione che "la faisait claudiquer" (84), il che implica un cont inuo zoppicare e non temporanei cedimenti: da notare che l'impersonale ce qu'il faut induce il lettore o la lettrice ad assumere una prospett iva di genere maschile. Da notare anche in questo brano la precisione fisiologica del francese e la strategia eufemistica dell'italiano. II motivo dell'atto sessuale compiuto a prescindere da una effettiva attrazione fisica motivata da qualità specifiche dei soggett i coinvolt i riappare a fine romanzo, quando il padre dell'io narrante prevalente, per obbedienza agli stereotipi albanesi secondo i quali il maschio deve avere una compagna, si accasa con una donna non più giovane $\mathrm{e}$ per nulla attraente, consumando il matrimonio solo per dimostrare la virilità tramite la prole. La ripugnanza nei confronti della deformità emerge con maggiore 
drammaticità nel testo francese: in „Ma è così brutta ... non potrei dormirci di più, faccio già letto a parte." (104) l'avverbio di quant ità più e quello di tempo già danno l'impressione di un processo che, nel soggetto maschile che in questo luogo prende la parola, si è svolto in un certo tempo, mentre "D'une laideur! II n'y aura pas de deuxième fois, je ne pourrais pas" (143) evoca una decisione repentina, accentuata dall'interiezione segnalata dal punto esclamativo, frutto di una presa di coscienza sdegnosa della bruttezza della compagna dopo il primo e unico rapporto sessuale: in una frase pare condensarsi una situazione decisamente grottesca che sembra invitare il lettore e la lettrice a condannare il comportamento dell'uomo. Prima di adeguarsi all'idea di doversi accontentare di un obbrobrio, il padre aveva cercato di usare la figlia per riavere (103) o più attivamente e, secondo la mentalità maschile rappresentata nel racconto, più onorevolmente reconquérir (142) la moglie. Nella narrazione dei tentativi di riavvicinamento alla moglie, la figlia viene metaforicamente designata tramite due oggetti diversi: come ponte (103) o come sauf-conduit (142), con una sfumatura attiva nella versione francese, in quanto essa stessa come salvacondotto si muove, mentre nella versione italiana come ponte dovrebbe consentire il movimento del padre verso la madre. Si ricordi che l'atto della fecondazione aveva avuto una inversa realizzazione linguistica col francese volto a rendere uno stato e l'italiano un processo, cosicché a livello di superficie il testo non appare coerente.

Il tentativo cosmetico messo in atto goffamente dal padre di una delle istanze narranti, che ci porta di nuovo agli oggetti nella loro materialità, tormenta l'elaborazione linguistica delle due versioni. Leggiamole una di seguito all'altra.

Ha i capelli completamente bianchi, i denti gli mancano. Faccio quello che mi sembra giusto fare: abbracciarlo. Anche lui mi abbraccia, io piango, lui piange. Mi rendo conto che piango per lo stupore del sentimento che provo; io non amo quest'uomo, il fatto mi provoca amarezza, mi duole, perché normalmente lo dovrei amare. Spontaneamente mi chiedo se le sue lacrime sono d'amore, mi domando questo vedendo quelle che verso io; non sono d'amore, sono fatte di colpevolezza di non amare, di disillusione sulla fedeltà dei sentimenti, di dispiacere profondo perché questo signore di fronte a me - mio padre biologico - è tutto coperto di capelli bianchi, l'ultima volta che l'ho visto erano ancora scuri (...) Oggi i suoi capelli sono nerissimi. Mi avvicino e ci diamo il buongiorno, ci abbracciamo. Le sue mani, le sue dita sono coperte di macchie nere. (98-99)

Ses cheveux ont ent ièrement blanchi, il n'a plus de dents. Je fais ce qu'il me semble juste de faire: je l'étreins. II m'étreint lui aussi. Je pleure, il pleure. Je me rends compte que, si je pleure, c'est de stupeur devant l'émotion que j'éprouve. Je n'aime pas cet homme, c'est un fait, et ce constat ne cause amertume et douleur, parce que il serait normal que je l'aime. Je me demande se ses larmes à lui sont des larmes d'amour; celles que je verse, après tout, n'en sont, elles sont faites de la honte 
que j'ai de ne pas l'aimer, de ma désillusion quant à l'impossible fidélité des sentiments humains et d'une déception profonde au spectacle de cet homme- mon père biologique a les cheveux blancs; la dernière fois que je l'ai vu, ils étaient châtains (...) Aujourd'hui, ses chevaux sont d'un noir d'encre. Je m'approche, nous nous souhaitons bonjour, nous nous embrassons. Ses mains sont couvertes de taches noires jusqu'aux ongles. (135-137)

Anzitutto osserviamo che mentre in italiano il padre ha un aspetto insolito, in francese attraverso il predicato blanchir si presenta in parte un processo; oltre, il colore di un tempo dei capelli in italiano è un vago scuro cui corrisponde un più preciso châtains; la pervasività della goffaggine nel maneggiare la tinta, in francese, è resa con jusqu(e) che precisa l'estensione della diffusione delle macchie, ma che potrebbe implicare una dimensione temporale; infine, nella versione italiana le macchie più genericamente coprono le mani. Nel leggere il prosieguo dell'incontro troviamo una conferma della tendenza alla maggior precisione della versione francese in quanto veniamo a saper che il padre, per riconquistare i favori della moglie, in italiano „si era colorato i capelli” (101), mentre in francese „il s'était teint les cheveux" (138); il predicato teint avrebbe potuto trovare il suo corrispett ivo in un più preciso e consueto tingere. Anche se dobbiamo ammettere che si sarebbe trattato solo di una differenza di registro senza rilevanti cambiamenti di significato, questo dato minimo sembra confermare la tendenza della versione francese all'innalzamento del registro linguistico. Quando padre e figlia sono a colloquio, il rapporto di precisione denotativa tra le due versioni s'inverte almeno nell'esito finale: alla versione italiana che suona "(lui) continua a parlare muovendo le dita e i palmi delle mani macchiati di nero" (100) corrisponde in francese "poursuit-il tout en remuant ses mains aux paumes et aux doits tachés" (138), in cui è presente la designazione analitica delle mani e delle loro parti, mentre è scomparsa la caratterizzazione cromatica. Poco oltre è il francese a riguadagnare in precisione in quanto alla seguente versione italiana "( $L$ )e sue mani stupide complici che non sanno nascondersi - dondolavano seguendo la sua voce: Quando sarai grande capirai che tua madre è talmente nervosa ..." (101) corrisponde il francese :

Ses mains - en complices parfaitement ineptes - se balançaient au rythme de sa voix, sans se soucier d'être vues: - Quand tu seras grande, tu comprendras que ta mère n'est qu'une boule de nerfs ... et que tant de choses sont de sa faute. (139)

Le mani, con personificazione di basso registro linguistico, sono "stupide" e "non sanno nascondersi", mentre in francese, con accostamento lievemente ossimorico, sono qualificate come "parfaitement ineptes”; inoltre, la vaga quant ificazione del nervosismo („talmente") in francese è rappresentata da un'iperbole che introduce anche l'attribuzione alla madre della responsabilità per i fatti verificatisi. Da notare che il testo presenta la visione della madre come un fascio di nervi senza fornire una voce o un punto di vista esterno che rilievi la visione di genere maschile e facendo 
passare l'idea che la madre sia, inequivocabilmente anche se metonimicamente, un fascio di fibre nervose: di nuovo fa capolino l'allusione alla riduzione dell'umano a element i fisiologici ineluttabili.

Se il negozio del padre e della figlia è ancora a venire, Ganimete e Bukuria, figlia e madre dedite al commercio sessuale, hanno potuto esercitare i loro affari nel discreto edificio in cui abitavano, che cosi viene presentato:

l'été venu, le lierre la sillonnait comme les veines le dos d'une main. La plante, rivée à la façade, recouvrait en partie une lucarne où, dans la pénombre, derrière les barreaux de fer, se détachait un petit visage. L'obscurité environnante, la pâleur diffuse de la jeune fille, le lierre qui l'enrubannait, cachant à demi sa chevelure, tout cela formait un mélange insolite et, d'une étrange manière, émouvant. (59)

Mentre in francese, giunta l'estate, l'edera assale la casa come le vene aggrediscono il dorso di una mano, in italiano, con cambiamento nell'aspetto temporale della predicazione, le vene assalgono la mano in piena estate:

L'edera l'assale come le vene una mano in piena estate. Avviticchiandosi attorno alla casa, la pianta ricopre parte della finestrella dove, nel buio nascosto dietro le sbarre di ferro contrastava un visino. Il pallore che si diffonde dalla ragazza, il nero della stanza attorno alla testa, l'edera che la orna celando una parte dei capelli inventano una mescolanza che incita un certo commuoversi inconsueto. (42)

Anche l'azione del rampicante è diversa. II testo francese, avviando la descrizione con l'immagine del dorso della mano, dà l'idea di un processo che, venendo, come l'estate, disegna sul dorso il percorso delle vene, ma poi ci presenta la pianta rivée, cioè inchiodata, con predicato che ha tra le sue possibili collocazioni anche prigionieri o forzati, indirettamente evocati dalla lucarne più un angusto lucernario o abbaino che un'idilica finestrella, dal quale si distacca il volto come se volesse liberarsi, potremmo aggiungere con forte gesto interpretativo; l'edera compie l'azione di infiocchettare o l'oscurità o il pallore della ragazza, cosicché per metonimia, si potrebbe intender che nasconda una parte dei capelli ornandoli e, cosi facendo, crea una mescolanza insolita e stranamente commovente. II testo italiano attacca in maniera più statica poiché siamo in piena estate (stato), ma l'edera non è inchiodata, dinamicamente si avviticchia alla casa; il carattere idillico che assume l'apertura col diminutivo finestrella è confermato dal diminutivo visino corrispondente a petit visage, che, nel caso in cui le due autrici avessero voluto togliere l'aspetto idillico del passo, avrebbe potuto avere come esito viso piccolo / minuto; la penombre si trasforma in un più deciso buio che acquista rilievo per la mancanza delle virgole a chiudere l'osservazione incidentale; il testo italiano riguadagna in dinamicità con il pallore che, invece di esser diffuso, si diffonde dalla ragazza, ma allo stesso tempo sembra schiacciare il quadro in una prospettiva bidimensionale attraverso il nero della stanza e 
i capelli, che, non rendendo chevelure con capigliatura attenua la potenziale corposità dei capelli; la chiusa contiene una marca decisamente personificante in quanto il quadretto non è solo commovente, ma incita un certo commuoversi. Gli elementi rilevati fanno pensare a una realizzazione linguistica della drammaticità della violenza di genere più intensa nel testo francese. Nella narrazione della vicenda di Bukuria e Ganimete si dà conto brevemente di ciò che succedeva alla figlia quando d'estate si stendeva al sole su uno sdraio. Nella versione italiana quasi non si accorgeva delle violente attenzioni del sole che "stuprava ogni poro della sua pelle scaldandola tutta" (45), mentre nella versione francese è lei stessa che sembra abbandonarsi al piacere: il predicato foviller, meno connotato dalla esplicita violenza dell'atto erotico, già incontrato proprio in un contesto di intrusione indesiderata da parte di maschi nel corpo delle donne, attrae l'azione in una zona che "le soleil la faisait fondre de plaisir, fouillant le plus petit pore de sa peau" (63) non farebbe supporre. Nelle due versioni si assiste a una sorta di naturalizzazione dei rapporti di genere molto prossimi alla violenza sessuale: il sole, con procedimento naturale e con meticolosa cura, stupra o fa irresistibilmente fondere di piacere frugando o tutti i pori, secondo la versione italiana, o i più piccoli pori, secondo quella francese, della pelle della ragazza; il testo francese, anche per l'effetto di accumulo di precedenti occorrenze del medesimo verbo fouiller, porta con più forza a interpretare i rapporti sessuali in una forma che nel testo vede la donna tendenzialmente passiva spettatrice di una meticolosa perquisizione e sottomessa a forze puramente istintuali. Dopo il trasferimento coatto delle due donne nell'istituto di correzione, la voce narrante si chiede che fine abbiano fatto. Nella versione italiana leggiamo quanto segue:

Per dei giorni ho cercato di scorgere tra i fili elettrici, questa volta senza lenzuola stese ad asciugare, il corpo di Ganimete, che non vedo più. Presa dall'angoscia della strada vuota del del suo tragitto, che mi agita lo stomaco, ho deciso che quando incontro Bukuria, malgrado quell'aria cosi severa per cui nessuno osa parlarle, io le voglio parlare, si che le voglio parlare. „Compagna Bukuria, non vedo più Ganimete, ha forse I'influenza?" (46)

La testo francese designa il surrogato per asciugare all'aria le lenzuola come nudo: visto che si parla di fili per stendere le lenzuola e non come di consueto di fili per la biancheria, la loro nudità può far pensare ai periodici denudamenti delle due donne che avrebbero come sfondo i rispett ivi letti per le prat iche ipocritamente condannate dalla morale della comunità di appartenenza, che, come visto, assume spesso il timbro della voce patriarcale:

Des longs jours passèrent, au cours desquels je tentai en vain d'apercevoir le corps de Ganimete entre les fils électriques, désormais nus. Je fus saisie d'angoisse, le chemin vide de son trajet me tordait l'estomac et je résolus, si jamais je croisais Bukuria, de lui parler. Malgré son air sévère qui dissuadait toute approche, je lui parlais, moi, bien sur 
que lui parlais. "Camarade Bukuria, je ne vois plus Ganimete. Est-ce qu'elle a attrapé la grippe?» (64)

A descrizione della cessazione del flusso di clienti in italiano leggiamo che „invece nessuno sfiora più la porta di quella casa" (46), mentre la versione francese ci dice che „mais la porte ne s'ouvrait plus pour personne" (64) che attraverso il predicato ouvrir riprende per opposizione col sostantivo mutisme la chiusura della bocca come strategia comportamentale: "Bukuria adopta le mutisme comme ligne de conduite. Elle n'ouvrait la bouche que pour les salutations d'usage et s'en tenait là." (61) In italiano manca il riferimento a una strategia coscientemente eletta e l'atto di parola viene espresso attraverso una soluzione sintattica, che, senza cambiare il significato della frase, afferma l'atto di aprire, anche se delimitato nei suoi contenuti qui esplicitamente nominati unitamente alla sottolineatura che non si andava oltre, constatando che non aveva altro da dire ma lasciando lettore e lettrice nell'incertezza sul significato da attribuire all'informazione poiché non é chiaro a chi vada attribuita e se si debba istituire un nesso causale tra i due segmenti connessi per asindeto: "Bukuria diventò muta con tutti, apriva bocca solo per un buongiorno e un buonasera, tutto finiva li, non aveva altro da aggiungere." (43) Anche in questo frammento delle vicende narrate le due soluzioni sembrano oscillare tra la volitiva assunzione di una postura (elle adopta) e un'ineluttabile legge di natura (diventó).

Si ricorderà che il padre della protagonista finisce in carcere. Ecco come viene narrato il fatto nella versione italiana:

Correva voce che fosse prigioniero. Sei mesi dopo la sua sparizione, lo venimmo a sapere. Era prigioniero politico. Perché la Questura minacciò la mamma? Loro sapevano dov'era il papà, che senso aveva questo interrogatorio? Cosa si aspettavano dalla mamma, dato che non era al corrente dell'accaduto? Misteri albanesi? II suo processo si tenne a porte chiuse. Non sapemmo mai di cosa fosse accusato. Dai discorsi sentiti a casa, anche il suo migliore amico aveva testimoniato contro di lui. (33)

"Misteri albanesi?": cosi è rubricata la procedura poi sommariamente descritta. La versione francese altera la distribuzione della materia e trasforma la riflessione dell'istanza narrante in uno scorcio dell'interrogatorio minaccioso da parte del personale della polizia:

La préfecture de police finit par appeler ma mère pour lui demander où était mon père : "Tu vas nous dire où se trouve ton mari, si tu ne veux être pendue par la langue!" Le bruit courut qu'il était prisonnier. Nous ne l'apprîmes réellement que six mois plus tard. Quant à savoir pourquoi l'Etat avait menacé ma mère, nous n'y parvînmes jamais. (47)

Questa versione dipinge ugualmente un'atmosfera di mistero che però non viene qualificata come tale e soprattutto acquista forza per il discorso diretto e per la prosopopea dello Stato e il categorico jamais che, unito al passato remoto del 
predicato parvenir, dà l'impressione di una definitiva chiusura, nonostante i presumibili reiterati tentativi di richiesta d'informazioni da parte della madre cui avevano fatto eco le minacce delle autorità. La personificazione dello Stato rafforza l'impressione di un mondo in cui i rapport i umani sono alienati da istanze opprimenti e, data la ripetuta naturalizzazione lungo tutto il testo di azioni che solitamente sono legate a soggetti dalle qualifiche personali, siamo portati a vedere anche nello Stato una forma di realtà ineluttabile di portata naturalistica. L'onnipresenza dello StatoPartito, che marxianamente si propone istanze di redenzione immediata degli oppressi, è tema ricorrente. Nella valutazione del potenziale contenuto ideologico di cartoline illustrate rappresentant i angeli, l'io narrante si confessa come segue:

lo non sapevo cos'erano gli angeli. Ma il partito e Dhoksi, loro lo sapevano. E con tutta evidenza non si amavano. L'aver portato le cartoline a scuola forni a Dhoksi l'alibi perfetto per disegnarmi miriadi di strisce di pelle bruciacchiata sulle cosce e sulla schiena. (21)

Con leggera variazione, il francese attacca con

(j)e ne savais pas que ces petites êtres étaient des anges. Mais le parti et Dhoksi le savaient, eux. Et, de toute évidence, ils ne s'aimaient pas. Ah, que de coups de verge brûlante m'ont-elles valus sur les fesses e les cuisses, ces cartes postales ! (41)

Anche in questo segmento l'istanza giudicante è, almeno in parte, delegata al Partito, cioè allo Stato, e le conseguenze sono rese più vaghe ma più intensamente vissute con l'indicazione dell'atto e l'interiezione, mentre la versione italiana presenta il fatto più dalla prospettiva del suo effetto con metafora straniante del disegno che contrasta col dato realistico della bruciatura. Da notare che qui l'italiano sembra esser più reticente rispetto al francese visto che in luogo di fesses ha schiena. Questa strategia appare in armonia con la vaghezza di cartoline in luogo del più preciso cartes postales, anche se in questo caso il francese non offre alternative, mentre l'italiano conosce la locuzione cartolina postale e suoi sottogeneri. La comparsa di un oggetto apparentemente marginale comunque genera una reazione di genere che assume più forte connotazione nel testo francese.

Fa di nuovo capolino lo Stato, del quale ci si libera solo con la morte, in occasione della sepoltura congiunta di una madre e delle ossa del figlio: questi era stato ammazzato per un amore oltre i conf ini etnici ammessi dal part ito e condannato dagli apparati di potere a una decomposizione per negata sepoltura; la pietà di un parente aveva recuperato il corpo e lo aveva messo in un'anfora in cui le ossa, macabra traccia oggettuale del figlio, erano state conservate fino al decesso della madre. Ecco il brano in italiano: "Li abbiamo lasciati poi in pace tutt'e due, madre e figlio, per scordare Madre-Partito. Sicuramente l'eternità non le sarà troppa." (77) A questo tenore di discorso la versione francese risponde come segue: "On les a enfin laissés tous deux, mère et fils, pour qu'ils oublient la Mère-Parti - l'éternité ne leur sera pas de trop." (107) Tra le due versioni in questo luogo c'é sintonia per quanto riguarda la 
prosopopea, mentre si può notare la maggior incisività del francese per l'assenza del sicuramente e per l'inequivocità del pronome soggetto di terza persona del verbo oublier: nell'italiano con la subordinata implicita, grammaticalmente, si dovrebbe attribuire l'azione al soggetto in prima persona plurale, vale a dire ai parenti, mentre secondo il senso la si potrebbe attribuire alla madre e al figlio; l'incertezza nell'uso dei pronomi si prolunga nella chiusa perché in italiano abbiamo la forma della terza persona singolare femminile mentre nel testo francese leggiamo la terza persona plurale.

La prigione in cui è rinchiuso il padre di cui abbiamo già discusso è collocata al nord del paese, dove si trovano montagne aguzze (38), la cui asperità del profilo è declinata nel francese arides (54) che implica piuttosto la scabrosità e soprattutto I'inutilizzabilità secondo una prospettiva antropica; alle montagne si ha accesso attraverso "quella strada pericolosa fatta di pietre e polvere" (38) che in francese, oltre a esser dangereuse, è fatta di più precisi cailloux ed è costeggiata di mauvaises herbes (54) che alludono a una sterilità che sconfina verso la dannosità con connotazioni antropomorfiche, visto che di erbe non adattate a scopi alimentari di solito si predica la tossicità la velenosità, ma non la malvagità. Di nuovo il testo francese presenta una personificazione tendente a cancellare i confini tra organico e inorganico che manca nella versione italiana. Anche la perquisizione delle due donne da parte di un'istanza pubblica presenta differenze:

Il poliziotto arrivò a controllare tutto quel che avevamo portato per papà. Fece a pezzi il pollo arrosto che la mamma aveva preparato, divise in quattro le pere per vedere ci avevamo nascosto qualcosa. Per farla breve, spappolò tutto il cibo: pronto per i cani. Ci spogliò e condusse una donna a controllare più int imamente me e mamma. (38)

è reso in francese con

Un policier nous y contrôla. Le poulet que maman avait rôti, il le coupa en petits morceaux, puis il divisa les poires en quatre afin de s'assurer que nous n'avions rien caché à l'intérieur. Bref, il réduisit tout en nourriture prête à être donné aux chiens. Ensuite il nous fit nous déshabiller dans une sorte de guérite où une femme fut appelée pour nus fouiller plus int imement. (53)

Nelle due versioni la violenza dell'apparato statale è distribuita in modo diverso: I'italiano indugia in un atto di violenza istituzionalizzata in cui il responsabile del carcere spoglia le due donne per poi affidare ipocritamente a una carceriera l'ispezione delle parti intime, mentre il francese, se, da un lato, attacca più discretamente lasciando che le due donne si svestano autonomamente, a meno che non siano spogliate da un'istanza non nominata, dall'altro, prosegue collocando l'ispezione in una garitte, cioè in uno spazio di chiara appartenenza militare e la carceriera è deputata a fouiller, che è espressione più forte rispetto al generico 
controllare e ci riporta agli sguardi indiscreti dei maschi e al sole che fruga i pori di Ganimete, confermando la direzione in cui collocare il predicato francese: istanze di diversa natura come sole, uomini e rappresentanti di un anonimo Stato-Partito sono dediti alla violazione della sfera del corpo proprio femminile ridotto a oggetto; vista la presenza tra le istanze or ora menzionate tanto di soggetti naturali quanto di soggetti umani, si è portat i a cancellare i confini tra animato e inanimato.

Come il mondo maschile, che possiamo ritenere esemplificato dal padre, appariva intento a cancellare processi di invecchiamento, forse causati dai maltrattamenti subiti in carcere, cosi il mondo femminile sembra esser assorbito da pratiche cosmetiche per catturare l'interesse dei maschi. Il livello elementare della cura della persona, vale a dire la pulizia, vede sostituzioni nei due testi che portano a due dimensioni sociali e culturali radicalmente diverse. Prendiamo le mosse da un segmento descrittivo dalle connotazioni idilliche:

Le domeniche sono popolate dagli andirivieni delle buone madri che preparano qualcosa da mandare al fornaio, chiacchiere sulla porta di casa, le ultime novità sul vicinato, io che lavo i vetri con dei pezzi di giornale, poi dovrò andare a comprare il petrolio per il fornello, la mamma fa la lavatrice e va a stendere i panni nel cortile. (44)

Le atmosfere qui evocate trovano nella versione francese espressioni nelle quali risuona un'eco proveniente da altri tempi o da altri ambiti culturali senza che ci sia spazio per pensare a scelte imposte dalla lingua: se „ma mère fait la lessive" significa che la madre usa pratiche per il lavaggio della biancheria ormai appartenenti al passato che comportano non solo tecniche ma anche spazi appositi, il testo italiano usa un elettrodomestico moderno, collocando cosi le azioni in spazi e tempi diversi. II testo italiano è meno marcato da attribuzioni di genere in quanto se si può immaginare un soggetto maschile alle prese con la lavatrice, lo stesso soggetto alle prese con liscivia offrirebbe un modello decisamente alternativo di mascolinità. Minore il divario tra i due testi nel caso del petrolio presente in entrambe ma che serve "pour le réchaud" (62) che forse non ha la stessa estensione semantica dell'italiano in relazione al fornello. Si ha l'impressione di una solo parziale metamorfosi nel paesaggio degli oggetti: la liscivia porta con sé una ricca serie di precise strutture materiali che non vengono toccate dall'ipotetica alternativa di réchaud e fornello. I due testi realizzano cosi in modo diverso la declinazione di genere delle strutture materiali.

Passiamo ora alla cosmesi in senso proprio. Nel romanzo di Vorpsi le donne vi si dedicano, ma i prodotti sono di bassa lega poiché, secondo la dizione italiana, si fa ricorso al "profumo del mercato nero" (90) mentre secondo quella francese e con indicazione dell'azione, il prodotto è "acheté au marché noir" (125). Di una giovane coppia si dice che "la loro unica stanza sapeva di lacca per capelli. Non si era ancora consumato il profumo che qualcuno gli aveva regalato per le nozze." (89) Se la versione italiana ammette la possibilità che prima o poi le essenze svaniscano, la 
versione francese sembra dare maggior persistenza ai due cosmet ici offerti agli sposi: "I'unique pièce où ils vivaient sent it la laque pour cheveux et le flacon de parfum qu'un invité leur avait offert le jour de noces." (124)

Il seguito della descrizione dall'abitazione degli sposi sposta l'attenzione sull'vomo, passando parallelamente dall'imperfetto al presente:

L'odore è fortissimo, il mal di testa assicurato, ma io ho un buon diritto a sopportare tutto ciò, secondo me ne vale la pena. Le labbra di suo marito di solito sono bagnate, anche se io non ho mai visto la sua lingua passare (fa parte dei tanti misteri che coprono la vita). (90)

Il francese conserva l'imperfetto marcando l'aspetto durativo insito in sopportare e di solito:

L'odeur était entêtante, la migraine garant ie, mais il y avait une raison à ce supplice qui me concernait et en valait la peine. D'ordinaire les lèvres du mari étaient humides, même si je n'ai jamais vu sa langue glisser sur elles (ce phénomène fait sans doute partie lui aussi des mystères qui étendent un voile sur la vie). (124)

Da rilevare che la versione francese ripete il significato testa lessicalizzato nel predicato e implicato nel disturbo fisico designato con precisione medica; e si noti che la secrezione salivare, che sembra far sbavare l'uomo, riconduce ancora una volta il desiderio maschile alla sfera degli ist inti, facendo pensare a uno slittamento della descrizione delle relazioni tra i sessi da dato culturale a dato naturale. Poche righe prima, della sposa si dice che "ama mettersi in testa un sacco di fermagli per i capelli, e riesce a tenerli sospesi come un nido d'uccelli quando il suo corpo acceca la vista con quei fiori enormi riempiti di colori forti." (89). II medesimo passo cosi suona nella versione francese: "Elle aime piquer ses cheveux d'une multitude d'épingles qui se sout iennent les unes les autres comme les brindilles d'un nid d'oiseau tandis que vous éblouissent les grandes fleurs aux couleurs éclatantes imprimées sur ses robes."(123) Come in passi precedentemente commentati, possiamo leggere anche qui un lieve innalzamento di registro linguistico dall'italiano sacco al francese multitude, e nella medesima direzione un acquisto in precisione per la menzione dell'oggetto su cui fanno bella mostra di sé i fiori sgargianti (robe); curioso notare che si può supporre un'influenza del francese sull'italiano nell'uso di ama in luogo del più usuale le piace, che sarebbe anche in maggior armonia col registro linguist ico presente nella versione italiana. Nel seguente giro di parole dalla stessa porzione di testo rileviamo espressioni fortemente animate:

Al posto delle sopracciglia, due righe nere di matita khol soggiornano sovrane alzandosi sempre più verso le tempie e, se mi ricordo bene, finiscono alle radici dei capelli o vi si inoltrano perdendosi. Sopra il labbro, che è sempre accuratamente rossissimo, un neo finto cerca di aggiungere qualche attratt iva a questa giovane donna che è sulla soglia dei trent'anni, o forse leggermente di più. (89) 
Se nella versione italiana le linee prendono dimora, il labbro sembra prendersi cura del proprio grado di rossore e il neo si occupa di rendere più attraente la donna, nella versione francese il resoconto è meno orientato verso inconsuete predicazioni:

A la place des sourcils, deux traits de khôl, noirs et souverains, s'étendent jusqu'au tempes et viennent mourir sous la racine de ses cheveux, à moins qu'ils ne s'y perdent. Au-dessus de ses lèvres, au fard rouge vif et sans défaut, un faux grain de beauté tente rendre plus attrayante encore cette jeune femme qui n'a pas trente ans, ou à peine. (123)

Un leggero slittamento si nota nel processo di applicazione del fard: nel testo italiano è l'intensità del colore che è accuratamente definita nel suo massimo grado tramite il superlativo assoluto, mentre in quello francese l'impeccabilità dovrebbe esser riferita al disegno delle linee, a meno che "sans défaut" non debba esser attribuito alla qualità della scelta - senza errore - tra diversi prodotti cosmetici. Rimane l'impressione di un'esagerata cura per l'aspetto esteriore sottolineata per iperbole dalla linea di mat ita che si prolunga fino alla radice dei capelli perdendovisi: l'immagine presenta in modo decisamente grottesco un mondo femminile dedito alla performance di una livrea accattivante cui corrisponde, come appena notato, una sorta di animalizzazione del comportamento maschile.

In una società dominata da appetiti sessuali che appaiono irresistibili e da una corruzione pervasiva, un cimitero, nel quale "i nostri piedi si trovano" (83) o al quale "nos pas nous conduisent", con prospettiva simile a quella di un automa scisso tra centro motore e arti, (116) $)^{25}$, nel capitolo dedicato a "Il dervish delle meraviglie", le tombe dei religiosi, che sembrerebbero costituire un'oasi di pace, sono tuttavia oggetto dello smascheramento dell'ipocrisia da parte di chi sa che "con i fiori di plast ica le tombe sono curate per più anni" (84). Un'ipocrisia maggiormente esplicata nella versione francese: "ces bouquets artificiels constituent une bonne excuse pour les familles, les tombes donnant l'illusion d'être entretenues pendant plusieurs d'années" (117). Delle tombe in italiano "non si vede neanche il nome" (83) mentre in francese si parla di "leurs épitaphes illisibles" (116), che, data l'illeggibilità, devono essere riconoscibili come epitaffi non per il loro contenuto bensi per la loro lunghezza, che quindi deve andare oltre lo spazio per un nome. Al neutrale "les plantes" (118) si sost ituisce "il verde che germoglia sul tuo cadavere" (84) con stridente contrasto tra la metonimia di tradizione letteraria che può risalire fino a motivi stilnovistici quali la verzura di Guinizzelli passando per il bel verde del "Ciabattino" di Saba, da un lato, e I'idea che quel verde sia nutrito di corpi in decomposizione, dall'altro lato ${ }^{26}$.

25 Frammentazione del corpo e trattazione delle sue parti come elementi autonomi fanno parte della poet ica di Vorpsi fin dal volume fotografico Nothing Obvious, Ginevra, Skalo, 2010 e da Vetri rosa

26 La tensione tra registro linguistico alto e designazione di un processo ad esso estraneo è attenuata se si tiene presente la rivalutazione delle sostanze in decomposizione presente nella chiusa ribadita della canzone '67 di F. De Andrè Via del campo: „dai diamanti non nasce niente, dal letame nascono i 
Concludiamo le nostre osservazioni sugli aspetti tematici coi dolciumi, che con la loro carica seduttiva ben si collocano in un mondo fatto di allettanti promesse cui i soggetti non riescono a offrire resistenza. "Lui si che aveva delle scatole piene di lokum” (27) o „il avait toujours des boîtes pleines de loukoums” (26). Si tratta del vecchio Babako, che per soddisfare le proprie voglie voyeurist iche e feticistiche, cioè per indurre ragazzine a mostrare "la farfallina", offre i preziosi dolciumi; tuttavia lo statuto degli oggetti nelle due versioni non è lo stesso. Nella prima frase attraverso l'avverbio di affermazione usato in forma enfatizzante risuona il confronto con altre persone che avevano lokum o semplicemente altri dolciumi o oggett i di attrazione per le bambine; la quantità di cui disponeva l'ottuagenario piega la curva prosodica verso l'esclamazione, mentre in francese si esprime che la dispensa era costante, senza per questo implicare il confronto con altri possessori dello stesso dolciume. Qualche riga oltre nel descrivere il dolciume la "noce ribelle che giocava a fare da bassorilievo" (27) è animata da un'intenzione ludica che é in linea con la figura, non del tutto inaspettata, del carattere riottoso del frutto in quanto richiama il sintagma ciuffo ribelle che potrebbe esser evocato dalla frequente presenza di profili umani nei bassorilievi, mentre in francese si legge un vezzo estetizzante da parte di un non menzionato ma presupposto fornaio o pasticcere: qui la noce è „posée là en guise de bas-relief." (26).

Due parole su fenomeni di ridondanza nell'area degli oggetti. Anzitutto "la profonde entaille infligée par le scalpel" (77): cosi si chiude la narrazione della prova sullo stato biologico del marito dell'insegnante di ginnastica, anche se poche righe prima, come nella versione italiana, si nominava il bistouri come strumento di verifica ${ }^{27}$. L'uso della variante scalpel toglie spessore al bistouri, ma ciò è compensato dall'uso di un termine che indica uno strumento proveniente da altro ambito delle pratiche umane, quello della dissezione che, se applicata all'essere umano, lo degrada ad oggetto inanimato o di interesse scient ifico: poiché la versione italiana ha solo il termine bisturi, manca in essa questo supplemento connotativo, il che appare in armonia con la soppressione dell'implicita sottolineatura sadica espressa dal participio passato infligée. Altra ridondanza in altro passo è il termine col quale si designa un elemento chimico che in normali condizioni termiche e di pressione si presenta sotto forma gassosa: il cloro (79), che tuttavia dobbiamo immaginare come

fior" (vv. 23-26) chiude un inno ad una vita colta anche nei suoi aspetti più sordidi. II riferimento alla canzone è pertinente non solo per la notorietà, ma anche per i temi: amore, prostituzione, transessualità, marginalizzazione.

La tensione tra registro linguistico alto e designazione di un processo ad esso estraneo è attenuata se si tiene presente la rivalutazione delle sostanze in decomposizione presente nella chiusa ribadita della canzone '67 di F. De Andrè Via del campo: „dai diamanti non nasce niente, dal letame nascono i fior" (vv. 23-26) chiude un inno ad una vita colta anche nei suoi aspetti più sordidi. II riferimento alla canzone è pertinente non solo per la notorietà, ma anche per i temi: amore, prostituzione, transessualità, marginalizzazione.

27 La differenza tra bisturi e scalpello anatomico è minima: il primo può avere lama intercambiabile, il secondo ha lama fissa e manico più robusto. 
parte di una molecola complessa perché, somministrato in soluzione nel tè, viene indicato come responsabile di un effetto soporifero sugli appetiti sessuali delle reclute maschili di una caserma per addestramento in cui si trovano anche donnesoldato. Due pagine oltre l'io narrante di sesso femminile si trova in infermeria dopo diciassette ore di un sonno senza sogno a causa dello sfinimento e della somministrazione di cloropromazine (81): ancora il cloro protagonista. Il testo francese al posto del cloro della prima occorrenza del testo italiano ha il bromure (110), più immediato perché indica per abbreviazione un composto chimico di uso per gli scopi cui si allude, mentre nella seconda mantiene il termine scientifico (112). Ciò che si guadagna in precisione, lo si perde in ispessimento semantico, perché probabilmente non tutte le lettrici e i lettori assoceranno con egual forza l'intervento sui processi fisiologici attraverso prodotti di sintesi diversi (chloropromazine e bromure). La terza ridondanza presente nel testo francese $(37 ; 40)$ riguarda la legna portata a scuola da discenti per il riscaldamento: in italiano se ne parla solo quando si menziona la punizione corporale cui è sottoposta la protagonista (20). I casi or ora commentat i non rappresentano dati irrilevanti perché denunciano in maniera sottile la pervasività di un apparato statale che, oltre ad offendere con strumenti da vivisezione, penetra nella fisiologia del corpo e nell'economia del focolare domestico.

Alcune lacune nel testo italiano attenuano la drammaticità delle relazioni umane. Il padre nella versione francese usa „une chambre à air (prélevée sur une carcasse de moto)" (45) per costruire un'arma con cui punire la figlia; la madre non appare meglio disposta nei confront i della ragazzina nonostante questa si prodighi per cucinare:

Juste avant qu'elle ne rentre du travail, je cuisinais des choses simples, comme des frites et des œufs frites. Nous en avons mangé des années entières. Ma mère était devenue très nerveuse (peut-être l'était-elle auparavant, mais maintenant je m'en rendais mieux compte). Elle me frappait souvent. (48)

La precisione della designazione dell'elemento propulsore per l'arma di offesa colloca l'attività in una sfera d'azione tipicamente maschile connotata secondo una dimensione di logoramento che ha ridotto uno strumento tecnico di locomozione a un corpo senza vita ${ }^{28}$. La madre, con cecità analoga a quella del padre, picchia la figlia senza che si sappia se utilizzi protesi o strumenti: da sottolineare ancora la naturalizzazione delle relazioni umane, visto che la nevrosi produce per semplice accostamento sintatt ico la punizione corporale.

28 Si può porre l'oggetto tra le categorie logoro-realistico e desolato-sconnesso sviluppate da Orlando ${ }^{2} 1994$. 


\section{Per una valutazione etica dell'auto-traduzione assistita}

Abbiamo prelevato dal testo di Vorpsi entità della vita materiale, che non sono cont inuativamente ascrivibili a soggetti agenti nelle vicende narrate. Negli oggetti selezionati abbiamo riscontrato una tendenza alla naturalizzazione dei rapporti sociali e in particolare di genere in modo non del tutto coerente sia all'interno delle strutture profonde che soggiacciono ai due testi sia, in maniera diversa, a livello di struttura di superficie nella formazione dei due testi, con pesi e connotazioni che oscillano tra naturalizzazione e personalizzazione. In armonia con la naturalizzazione di strutture sociali, che cosi conferiscono una dimensione deterministica al rapporto col soggetto storico, sembra stare uno Stato, quello albanese in un certo momento storico assunto a emblema di una condizione esistenziale, che promette redenzione ma offre meccanica applicazione di un materialismo rozzo. Ne esce un'immagine desolante dell'umanità, del mondo maschile e della proiezione sulla donna delle aspettat ive dei maschi, senza che quest'ult ima mostri chiare marche di dissidenza. La polarizzazione tra i due generi non è irrilevante perché crea gruppi di oggetti e posture nei loro confronti omogenei anche se in parte sovrapposti: si pensi ai cosmetici.

L'opera di traduzione implica sempre un adattamento di un testo che deve esercitare la sua intentio operis sul lettore o sulla lettrice di una lingua diversa e di un contesto culturale potenzialmente distinto rispetto a quella in cui il testo è stato scritto: quali siano le leve sulle quali il traduttore o la traduttrice e l'auto-traduttore o la auto-traduttrice mettono mano è lasciato alla scelta dei soggetti coinvolti e la loro libertà è tradizionalmente tanto più ampia quanto maggiore risulta il coinvolgimento dell'istanza autoriale nell'att ività traduttiva. Tuttavia in una prospett iva di genere nel caso da noi analizzato pare di dover rilevare un'oscillazione nell'attribuzione delle cause di una situazione di sottomissione della donna a condizioni di esistenza alienant i determinate dalla struttura patriarcale: in entrambe le versioni e con diverse distribuzioni, in parte la donna pare partecipe delle strutture di potere che ne determinano la qualità della vita e delle relazioni umane, in parte pare soffrire per ciò in uno stato di totale impotenza. Nell'organizzazione della materia che soggiace ai due testi sembra mancare una marca che apra verso orizzont i crit ici di redenzione nei confronti della situazione di alienazione del soggetto femminile. Una pendolarità di fondo disorientante ${ }^{29}$ pare esser attiva nelle diverse strategie che dominano la costruzione dei due test i portando a pesi diversi di figure della naturalizzazione come la personificazione, la metonimia e la metafora e a esiti stilistici di altra natura ugualmente non univoci nel connotare le relazioni di genere. Come lettori e, forse a maggior ragione, come lettrici proviamo disorientamento per due versioni di una

29 Decisamente positive sono invece le caratterist iche di pensatori e pensatrici pendolari analizzati da Rigotti 2006: la definizione di cosa faccia di un soggetto pendolante un'istanza critica esula dalle presenti note. 
materia narrativa che appare come denuncia della discriminazione femminile e allo stesso tempo priva di marche che consentano di vedere posture schierate sul fronte femminile o su quello della relazione di genere: riprendendo la distinzione di Shread, possiamo affermare che lo statuto ambiguo del testo in auto-traduzione assistita di Vorpsi non appare costruito su un'attitudine formativa coerente che contribuisca a stabilizzare un discorso di fondazione dell'ident ità ${ }^{30}$. Se questo è l'intento dell'autrice nelle due versioni della medesima materia, ci chiniamo alla sua volontà, ma nel confronto con l'intenzione sedimentata nel testo ci permettiamo di esprimere la nostra insoddisfazione di lettori e lettrici che esercitano attenzione nei confronti di una coerente declinazione di genere tanto sul piano informativo quanto su quello formativo.

\section{Conclusioni}

Dall'osservatorio di un'analisi di pratiche traduttive che cerchi un rapporto dialettico tra strutture di superficie e strutture profonde, anche se non si condividesse la conclusione cui siamo giunti nel caso specifico, ci pare comunque di poter sostenere che l'analisi comparata, pur orientata alla focalizzazione su un aspetto ristretto come la menzione di oggetti, abbia consentito di lumeggiare passi problematici attraverso le frizioni tra le due versioni, cosicché la procedura potrebbe esser utilizzata in casi simili per scandagliare le potenziali strutture profonde di testi di traduzione, a prescindere dal fatto che si present i nella forma dell'auto-traduzione, della traduzione o di una forma intermedia come l'auto-traduzione assist ita.

\section{Bibliografia}

Amati Mehler J., Argentieri S., Canestri J. (1900). La babele dell'inconscio. Milano, Italia: Cortina.

Arrojo, R. (21992). Oficina da traducão - A teoria na práctica, São Paulo, Brasile: Àtica.

Babel, R. (2015). Translationsfiktionen. Bielefeld, Germania:Transkript.

Bachmann-Medick, D. ('2009). CulturalTurns. Amburgo, Germania: Rowohlt.

Baker, M. (Ed.). (1998) Routledge Encyclopedia of Translation Studies. Londra - New York, Gran Bretagna - USA: Routledge.

Borek, J. (1998). Der Übersetzer ist weiblich und damit unsichtbar. Übersetzen als ein Herrschaftsverhältnis, unter anderen. in Quo vadis Romania?, 7, pp. 27-33.

Borutti, S., Heidemann U. (2012). La Babele in cui viviamo. Traduzioni, riscritture, culture. Torino, Italia: Bollat i Boringhieri.

Chamberlain, L. (2000). Gender and the Metaphorics of Translation. Venuti, L., (Ed.) The Translation Studies Reader, Londra - New York, Gran Bretagna - USA: Routledge, pp. 314-330.

30 Sulle potenzialità e i limiti della traduzione per la costruzione del sé si veda Resch, 2001. 
Cordingley, A. (2013). Self-Translation: Brokering Originality in Hybrid Culture, London, Gran Bretagna: Bloomsbury Publishing.

Courtés, J. (1991). Analyse sémiot ique du discours. Parigi: Hachette.

Forster, L. (1970). The Poet's Tongues. Multilingualism in Literature, Cambridge, Nuova Zelanda: Cambridge University Press e University of Otago Press (New Zealand).

Furster Ortuño, À. (2008). Concepte d'equivalència: gènere i traducció. in Camps, A. Zybatow, L. (Eds.) Traducción e interculturalidad, Vienna, Austria: Peter Lang, pp. 141-155.

Grutman, R. (2008). Self-translation. in Baker M., S.aldahna G. (Eds.). Routledge Encyclopedia of Translation Studies. London, Gran Bretagna: pp. 257-26o.

Guldin, R. (2004). Translation, Self-Translation, Retranslation. Exploring Flusser's Multilingual Writing-Practice. in Id. (Ed.) Das Spiel mit der Übersetzung, Tubinga, Germania: Francke, pp. 99-118.

Hirsch, A. (1997). Die geschuldete Übersetzung. in Id. (Ed.) Übersetzung und Dekonstruktion, Francoforte s.M., Germania: Suhrkamp, pp. 396-426.

Hokenson, J. W., Munson, M. (2007). The bilingual Text. History and Theory of Literary Self-Translation. Londra - New York, Gran Bretagna - USA: Routledge.

Kappeler, A. (2008). Berliner Kindheit - Enfance berlinoise. Verfahren einer Selbstübersetzung Benjamins. Università di Vienna (Dissertazione).

Mayanja, S. (1999). "Pthwoh" Geschichte, bleibe ein Zwerg, während ich wachse". Untersuchungen zum Problem der Übersetzungen afrikanischer Literatur ins Deutsche. Hannover, Germania: Revonnah.

Monti, J. (2011). Scrittura, traduzione e ipertesti: voci al femminile. in Palusci O. (Ed.) Traduttrici. Female Voices across Languages, Trento, Italia: Tangram Edizioni, pp. 321-332.

Munday, J. Introducing Translation Studies. Londra, Gran Bretagna: Routledge, 2001.

Orlando, F. ('1994), Gli oggetti desueti nelle immagini della letteratura. Torino, Italia: Einaudi.

Palusci, O. (2010). Per una declinazione della voce traduttrice. in Ead. (Ed.) Traduttrici. Questioni di gender nelle letterature di lingua inglese. Napoli, Italia: Liguori, pp. IX-XVIII.

Râbacov, G. (2013). Self-Translation as Madiation between cultures, in International Journal of Communication Research, 3, 1, pp. 66-69.

Resch, R. (2001). Übersetzen als Empowerment. in Wolf, M., Messner S. (Ed.). Übersetzung aus allen Fraven Länder. Graz: Leykam, pp. 119-126.

Ricoeur, P. (2004). Sur la traduction. Parigi, Francia: Bayard.

Rigotti, F. (2006). Il pensiero pendolare. Bologna, Italia: Il Mulino.

Shread, C. (2011). On Becoming in Translation: Articulating Feminisms in the Translation of Marie Vieux-Chauvet's Les rapaces. in von Flotow L. (Ed.), Translating Women, Ottawa, Canada: University of Ottawa Press, pp. 283-303. 
Simon, S. (1996). Gender in Translation. London - New York, Gran Bretagna - USA: Routledge.

Snell-Hornby, M. (2001). Übersetzungswissenschaft im Aufbruch. Frauen als Vordenkerinnen neuer Perspektiven, in Wolf M., Messner S. Übersetzung aus allen Fraven Länder. Graz, Austria: Leykam, pp. 23-30.

Snell-Hornby M., Hönig H. Kußmaul P., Schnidt P. (1998). Handbuch Translation. Tubinga, Germania: Stauffenburg.

Spivak, G. C. (2000). The Politics of Translation. in Venuti L., (Ed.) The Translation Studies Reader, Londra - New York, Gran Bretagna - USA: Routledge, pp. 397416.

Stolze, R. ( $\left.{ }^{3} 2001\right)$. Übersetzungstheorien. Eine Einführung. Tubinga, Germania: Narr.

von Flotow, L. (2001). Genderkonzepte im Wandel. in Wolf M. e Messner S. (Ed.). Übersetzung aus allen Fraven Länder. Graz, Austria: Leykam, pp. 49-62.

von Flotow, L. (2010). Gender in Translation. in Gambier Y., van Doorslaer L. (Ed.) Handbook of Translation Studies, V. 1, Amsterdam, Olanda: J. Benjamins Company, pp. 129-133.

Vorpsi, O. (2012). Lenzuolo bianco. in AAVV, Ti racconto una cosa di me. Scritture e fotografie da collezioni private. Palermo, Italia: Edizioni di passaggio, pp. 22-23.

Vorpsi, O. (2006). Il paese dove non si muore mai. Torino, Italia: Einaudi.

Vorpsi, O. (2004). Le pais où l'on ne meurt jamais. trad. di Pozzoli M. con la collaborazione dell'autrice, Arles, Francia: Actes Sud.

\section{Cómo referenciar este artículo/How to reference this article:}

Rossi, M. (2018). Gli oggetti di una voce femminile al vaglio dell'etica dell'auto-traduzione assistita: il paese dove non si muore mai di Ornela Vorpsi. iQUAL. Revista de Género e Igualdad, 1, 159-189, doi: 10.6018/iQual.301191

Rossi, M. (2018). Gli oggetti di una voce femminile al vaglio dell'etica dell'auto-traduzione assistita: il paese dove non si muore mai di Ornela Vorpsi. [A female voice on the examination of the ethic of assisted self-translation: Ornela Vorpsi's il paese dove non si muore mai]. iQUAL. Revista de Género e Igualdad, 1, 159-189, doi: 10.6018/iQual.301191 